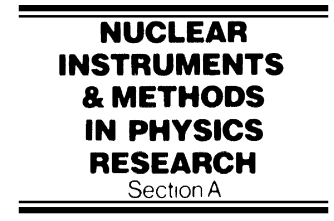

www.elsevier.nl/locate/nima

\title{
Monte Carlo simulation of the X-ray response of a germanium microstrip detector with energy and position resolution
}

\author{
G. Rossi*, M. Sanchez del Rio, P. Fajardo, J. Morse \\ European Synchrotron Radiation Facility, BP 220, F-38043 Grenoble-Cedex, France
}

Received 18 May 1998; received in revised form 18 February 1999

\begin{abstract}
We present Monte Carlo computer simulations of the X-ray response of a micro-strip germanium detector over the energy range 30-100 keV. The detector consists of a linear array of lithographically defined $150 \mu \mathrm{m}$ wide strips on a high purity monolithic germanium crystal of $6 \mathrm{~mm}$ thickness. The simulation code is divided into two parts. We first consider a $10 \mu \mathrm{m}$ wide X-ray beam striking the detector surface at normal incidence and compute the interaction processes possible for each photon. Photon scattering and absorption inside the detector crystal are simulated using the EGS4 code with the LSCAT extension for low energies. A history of events is created of the deposited energies which is read by the second part of the code which computes the energy histogram for each detector strip. Appropriate algorithms are introduced to account for lateral charge spreading occurring during charge carrier drift to the detector surface, and Fano and preamplifier electronic noise contributions. Computed spectra for different energies and beam positions are presented and compared with the experimental results obtained at the European Synchrotron Radiation Facility (ESRF), where the detector was scanned with a micro-beam of monochromatic photons. The remarkable agreement between the computed and measured data is discussed, and we show that consideration of charge diffusion and the resultant signal splitting between adjacent strips is essential to explain the observed results. We also comment upon the relevance of phenomena neglected in our model, such as secondary electron transport and charge trapping. (C) 1999 Elsevier Science B.V. All rights reserved.
\end{abstract}

Keywords: Monte Carlo; Germanium; Detector; Microstrip; X-ray; Diffusion; Charge; Splitting

\section{Introduction}

This simulation work was carried out in the framework of a detailed characterisation of the energy and spatial response of germanium microstrip detectors over the range $10-100 \mathrm{keV}$ per-

\footnotetext{
* Corresponding author. LASSP, Cornell University, 192 Clark Hall, Ithaka, NY 14853-2501, USA. Tel.: 607-255-8678; fax: 607-255-8751.

E-mail address: gr32@cornell.edu (G. Rossi)
}

formed at the ESRF, for which experimental results from prototype detectors have been previously reported $[1,2]$. A monochromatic synchrotron X-ray beam of $15 \mu \mathrm{m}$ FWHM was used as a probe and scanned in $10 \mu \mathrm{m}$ steps across the detector surface. For each beam position, the energy responses of the individual strips of the detector were measured and histograms of their corresponding spectra computed. Large distortions were observed in the experimental spectra when the scanning beam was incident on the isolation gaps between neighbouring strip contacts. In this paper we show that these 
distortions are explained by considering that two adjacent strips contribute in parallel to the collection of the charge created by each photon absorption event. This effect, which we refer to as charge splitting, results from charge diffusion which increases the apparent size of the charge cloud created inside the germanium crystal at the point of photon absorption. Using a Monte Carlo simulation code with no free parameters we demonstrate that energy spectra for a micro-strip germanium detector can be generated which accurately represent the experimentally obtained results.

\section{Detector description}

The detector tested at the ESRF is a p-i-n type, consisting of 28 linear strips lithographically defined by boron implantation on a $6 \mathrm{~mm}$ thick, high purity p-type germanium crystal. Each strip is $20 \mathrm{~mm}$ long and $150 \mu \mathrm{m}$ wide and forms an Ohmic contact with the germanium substrate, with electrically isolating gaps of $50 \mu \mathrm{m}$ width separating the adjacent diode strips. A rectifying contact exists between the substrate and a continuous lithium diffusion which forms the back-side electrical contact. The segmented-contact, strip side of the detector is exposed to X-rays. Schematic representations of the detector geometry are shown in Figs. 1a and $\mathrm{b}$. The germanium crystal is maintained in a vacuum cryostat at $\approx 80 \mathrm{~K}$ by means of a cold finger in contact with liquid nitrogen. Charge preamplifiers are located externally to the cryostat with low capacitance connections made to each detector strip. The measured energy resolution of $1.4 \mathrm{keV} \mathrm{FWHM} \mathrm{(} 2 \mu$ s pulse shaping time) is dominated by the room temperature charge preamplifier electronic noise [1,2].

\section{The simulation software}

The modelling of the interactions between the photon beam and the detector was performed using Monte Carlo simulations with the EGS4 software package [3]. The standard EGS4 core was complemented with the Low-Energy Photon-Scattering Expansion for the EGS4 Code (LSCAT) developed
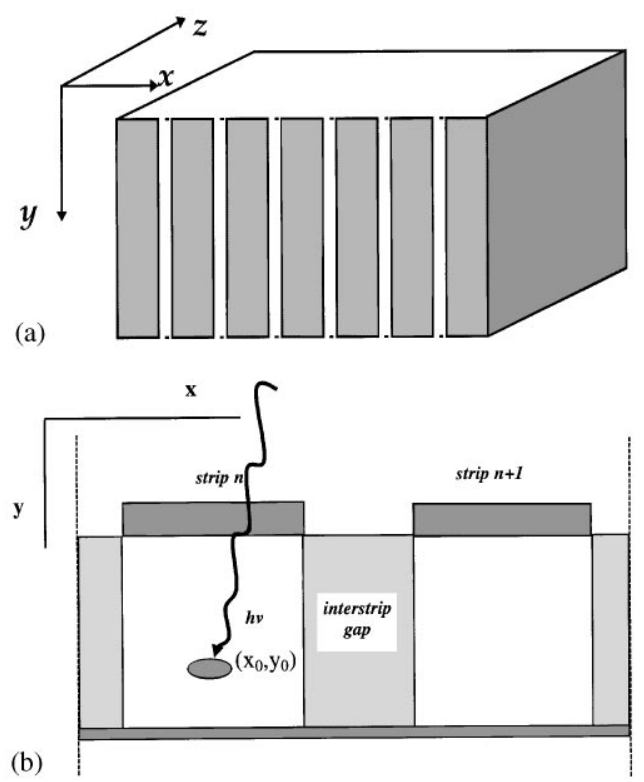

Fig. 1. (a) Schematic of the germanium detector showing the coordinate system used for the simulation code and (b) crosssection in the $x, z$ plane of two adjacent contact strips. The detector is assumed to have infinite dimension along the $y$-axis.

by Namito et al. [4]. This includes Doppler broadening of Compton-scattered photons [5]; linearly polarised photon scattering [6]; fluorescence shells; and low-energy photon transport. Photoelectric, Compton, and Rayleigh scattering processes were all considered in the simulation code, but the effect of electron transport, i.e. the finite range of energetic electrons slowing down in the germanium bulk, was ignored. The significance of electron transport in the absorption of X-rays at high energy was determined in a second set of simulations described in part $\mathrm{V}$. The simulation comprises two well-defined parts.

\subsection{Part I}

The history of the physical interactions of an ensemble of photons is computed. A $10 \mu \mathrm{m}$ wide monochromatic photon beam in normal incidence on a $6 \mathrm{~mm}$ thick, laterally infinite Ge slab is defined within the EGS4 code. The photons are generated using a Monte Carlo technique that samples spatially the beam profile which was measured 
experimentally. Each incident photon is tracked by the EGS4 code until it is totally absorbed or it has escaped from the detector volume. A parameter set that describes each interaction event inside the detector is recorded to a file. This parameter set includes the spatial coordinates of the interaction point(s), the amount of the deposited energy (s) and the nature of the interaction(s) (Photoelectric, Compton or Rayleigh). An identifier is also recorded to associate each interaction with its parent photon, because several interactions may occur before either the complete absorption of the incident photon or its partial loss from the detector volume. The fluorescence K- and L-shell photons created in the interactions are also tracked until their total absorption (or escape) and their histories added to the parent photon record. To obtain results with the statistical variance similar to that of the experimental data, about 50000 incident photons are scored, with a computing execution time of about $15 \mathrm{~s}$ in an HP 750 Unix workstation.

\subsection{Part II}

The second part of the simulation code uses the file generated by the EGS4/LSCAT code which contains the list of photon histories to construct histograms of energy deposited in each of the nine adjacent detector strips. The history file is processed as follows:

(a) Conversion of the absorbed photon energy into free charge. The EGS4/LSCAT code provides a three-dimensional map of the deposited energy inside the detector for each interacting photon. This is converted to an equivalent free charge carrier map by simple scalar division between the deposited energy and the average energy absorption of the germanium crystal per electron-hole pair creation. We thus implicitly assume that electrons generated by any Photoelectric or Compton interaction are completely absorbed locally at their point of creation; that is, electron transport is neglected. As demonstrated in Part V, the effect of electron transport on the spatial distribution of the deposited energy is important only for high-energy incident photons: note that at photon energies below $100 \mathrm{keV}$, the created free charge is contained within a sphere of radius $<25 \mu \mathrm{m}$ [7], i.e. less than the width of the inter strip isolation gap of the detector studied here.

(b) Charge collection from the germanium bulk to the strips. The charge collection in the detector volume is represented by a simple geometrical model, in which a strip collects only the charges created in the projected volume beneath it. Complete charge collection is assumed from within the detector volume, that is, we assume no charge loss mechanisms such as recombination or trapping. To account for charge splitting between adjacent strips arising from diffusion occurring as the charge cloud drifts to the front surface of the detector strip, the initial spatial distribution of free charges computed for each impinging photon by the EGS4 code is pre-processed with an appropriate charge spreading algorithm, as explained below. In the simulation code a virtual X-ray beam is scanned across the detector strips in a manner similar to that of the experimental measurement, and the detector response computed for all beam positions across the detector contact strips and the interstrip isolation gaps.

(c) Two noise components, both considered to have Gaussian distributions, are added in quadrature with the charge signal. The first (Fano noise) corresponds to the statistical fluctuations in the generation of charge carriers at the point where energy is deposited. Its standard deviation is equal to $\sqrt{(F \omega E)}$, where $F$ is the Fano factor, $\omega$ is the average energy per electron-hole pair creation (respectively, 0.12 and $2.96 \mathrm{eV}$ for germanium at $77 \mathrm{~K}$ ), and $E$ is the photon energy absorbed [8]. The second noise component is the read-out noise of the system electronics (dominated by the $0.6 \mathrm{keV}$ root-mean-square preamplifier noise). A random noise value weighted according to the total Gaussian noise distribution and with a random sign is added algebraically to the total energy from charge originating from the same parent photon collected under a single strip. Finally, the simulation code accounts for the non-linearity of energy response of the detector system (which was experimentally determined to be at a $\sim 1 \%$ level, arising from the complete signal processing electronic chain).

The code described above, together with its graphical user interface, are written in the IDL 
language [9] and execute on an HP 750 Unix workstation in less than $1 \mathrm{~min}$.

\subsection{Charge splitting algorithm}

The charge splitting algorithm is applied to the photon interaction histories computed by the EGS4 code. The initial free charge carrier cloud generated by a photon absorption process is assumed to have zero spatial dimension (charge transport is ignored). However, after its creation in the semiconductor bulk, the charge cloud undergoes thermal diffusion during its drift to the collecting strip electrode. It has been shown [10] that during the drift time the increasing charge spatial distribution follows a Gaussian profile $q(x)$, as a function of the drift distance. The integral under the Gaussian corresponds to the number of carriers generated by the photon absorption if charge loss effects are neglected, which is an excellent approximation for high purity germanium detectors. The standard deviation $\sigma$ of the Gaussian profile of charge is expressed by [10]:

$\sigma=\sqrt{2 D_{\mathrm{p}} t}$

where $D_{\mathrm{p}}$ is the diffusion coefficient and $t$ is the drift time inside the detector volume. $D_{\mathrm{p}}$ is computed according to the Einstein relationship $D_{\mathrm{p}}=\mu_{\mathrm{p}}^{k_{\mathrm{B}} T} / q$, where $\mu_{\mathrm{p}}$ is the mobility of the charge carrier, $k_{\mathrm{B}}$ is the Boltzmann constant, $T$ is the temperature, and $q$ is the carrier charge. Eq. (1) can be written as

$\sigma=\sqrt{2 \mu_{\mathrm{p}} \frac{K_{\mathrm{B}} T}{q} \frac{z}{\mu_{\mathrm{p}} E}}=114.9 \sqrt{\frac{z d}{V}}(\mu \mathrm{m})$

where $E$ is the electric drift field inside the detector germanium bulk; $d$ is the active detector thickness $(5 \mathrm{~mm})$; and $z$ is the elapsed drift distance from the point of charge generation. This distance is assumed to correspond to the depth of the photon interaction. The electric field inside the detector is considered uniform, a reasonable assumption for the planar geometry detector which was operated in a heavily overdepleted condition (the applied bias potential $V$ was $500 \mathrm{~V}$ while the detector was fully depleted at $\approx 60 \mathrm{~V}$ ). Eq. (2) provides the projected cloud size at the detector surface for a charge created at a distance $z$ inside the detector volume.
Note that the extent of carrier diffusion is independent of the detector material and depends only on the photon interaction depth, temperature and applied electric field.

As the detector strip length was much greater than the strip width, the detector can be considered infinite along the strip axis and the detector geometry reduced to two dimensions. The 'centre of gravity' of the charge profile at the surface is then simply given by the photon interaction coordinates $x_{0}, y_{0}$. We compute the fraction of charge collected by each detector strip when the free charge carrier cloud reaches the germanium surface as the integration of the Gaussian profile as described by Eq. (2) over a domain equal to the strip width. As the strip width is much larger than the typical diffusion length, only the two neighbouring strips nearest to the photon impact point $x_{0}, y_{0}$ need to be considered. The fractions of charge deposited on the left $(\mathrm{L})$ and on the right $(\mathrm{R})$ strips are calculated as

$Q_{\mathrm{R}}=\int_{0}^{+\infty} q(x) \mathrm{d} x$ and $\quad Q_{\mathrm{L}}=Q-Q_{\mathrm{R}}$

where

$Q=\int_{-\infty}^{+\infty} q(x) \mathrm{d} x$

and the origin $x=0$ is taken at the line $a-a$ ' midway between the two adjacent. This is shown schematically in Fig. 2 where the centre of gravity of the

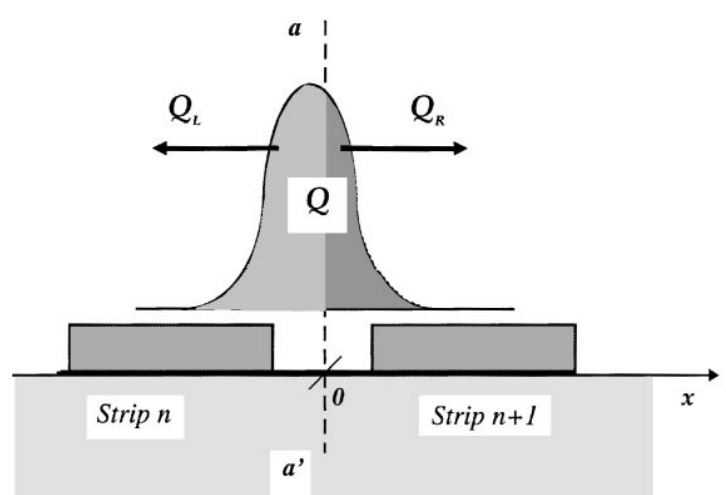

Fig. 2. Schematic of the charge splitting mechanism. This particular case shows the larger fraction $Q_{\mathrm{L}}$ collected by the left strip. 
charge distribution is set toward the left-hand strip: in this example the charge cloud will be split with the larger fraction collected by the left-hand strip, $Q_{\mathrm{L}}$.

\section{Results}

We present simulated detector spectral energy responses for two different situations:

(i) beam incident on contact strip centre: energy spectra are computed for beam energies of 35 , 60 and $100 \mathrm{keV}$

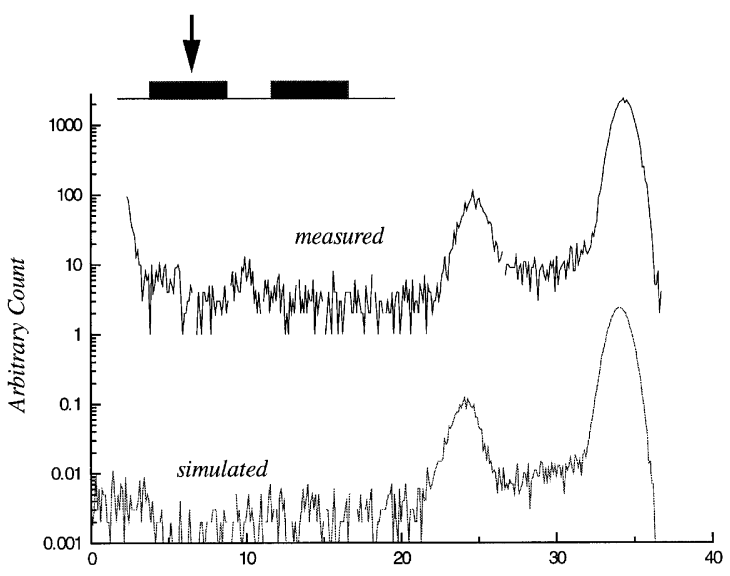

(a)

Energy $(\mathrm{KeV})$ (ii) beam scanned across the interstrip gap: a virtual $60 \mathrm{keV}$ beam is scanned in step increments of $10 \mu \mathrm{m}$ from the contact strip edge across the interstrip gap.

\subsection{Beam incident on contact strip centre}

Simulated energy spectra are shown in Figs. $3 \mathrm{a}-\mathrm{c}$, respectively. At 35 and $60 \mathrm{keV}$ the agreement between the experimental and calculated spectra is very good, with both the full energy photo-peak and the unresolved germanium $\mathrm{K}_{\alpha}, \mathrm{K}_{\beta}$-shell fluorescence escape peaks at $\approx 10 \mathrm{keV}$

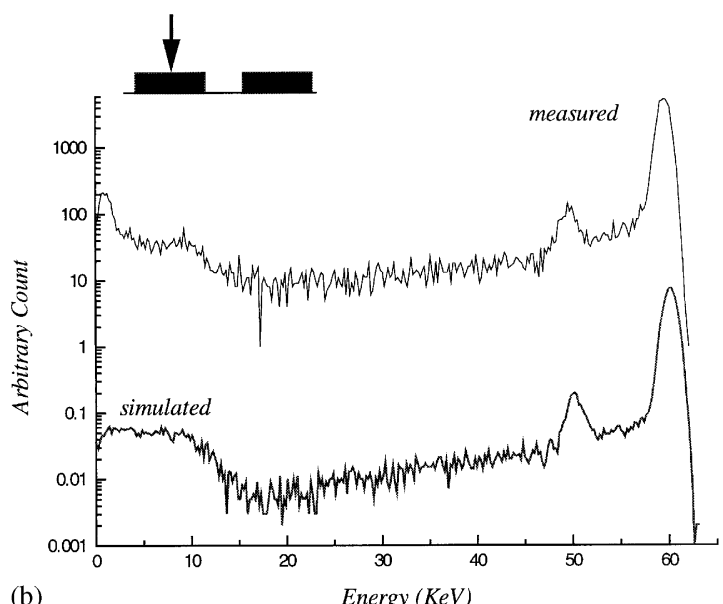

(b)

Energy $(\mathrm{KeV})$

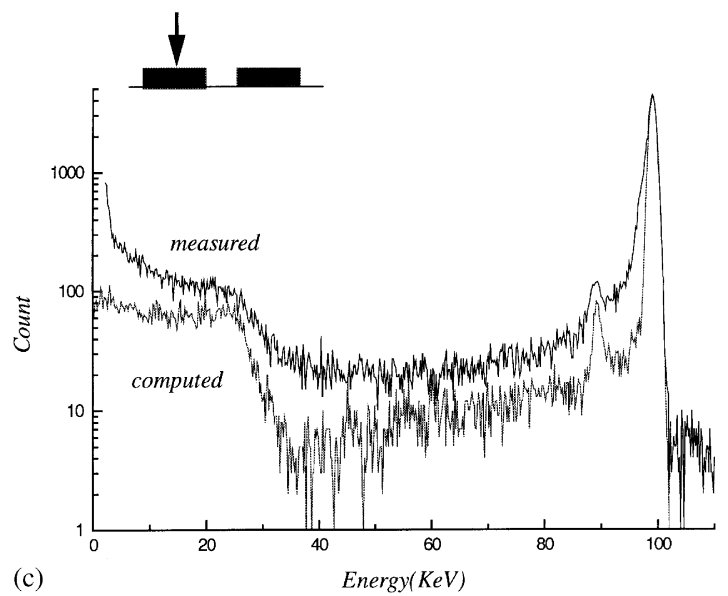

Fig. 3. Computed and simulated spectra for incident photon beams at energies (a) 35, (b) 60 and (c) $100 \mathrm{keV}$, respectively. The beam is incident on the contact strip centre. Experimentally measured data are shown in the top curves. For clarity, the simulated spectra of Figs. $3 \mathrm{a}$ and $\mathrm{b}$ are multiplied by $10^{3}$. Arrows indicate the beam position with respect to contact strips. 


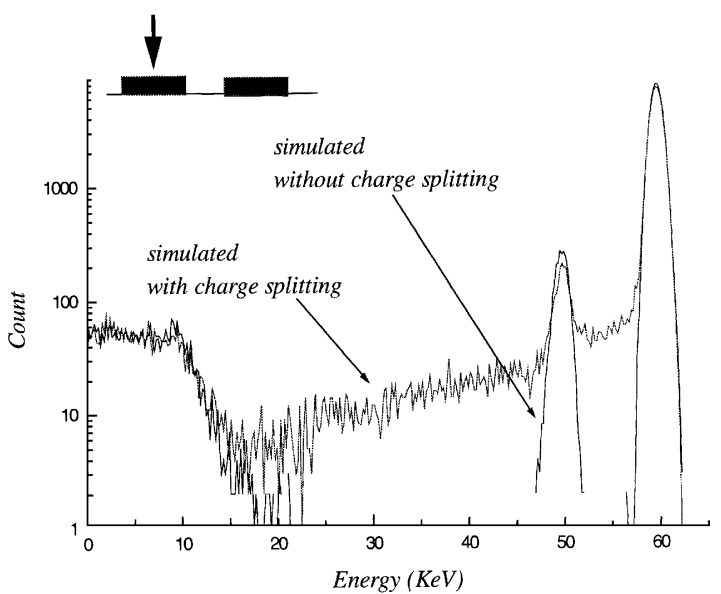

Fig. 4. Simulated spectra for an incident photon beam of $60 \mathrm{keV}$ incident on the strip centre obtained both with and without use of the diffusion charge splitting algorithm.

below the photopeak correctly reproduced. The $\mathrm{K}$-shell escape peak feature arises from the significant fraction of fluorescence photons which escape from the detector surface. Computed spectra at 35 and $60 \mathrm{keV}$ show the tailing behaviour which arises from charge splitting, in excellent agreement with that obtained by experiment. For $60 \mathrm{keV}$ incident beam energy, the computed low-energy Compton continuum which arises from the Compton recoil electron energy distribution matches that measured experimentally both in shape and intensity. The Compton edge at $11.4 \mathrm{keV}$ is also correctly reproduced.

In Fig. 4, the simulated spectrum at $60 \mathrm{keV}$ is compared to that produced with the charge splitting routine disabled (in this case, only the photon absorption processes contribute to the spectrum as the charge created in the detector volume is simply projected perpendicularly onto the detector surface with no charge diffusion). The spectrum produced with charge splitting switched off shows no lowenergy tailing or background, but presents the other features related to the photon absorption processes (escape peak and Compton continuum). This clearly indicates the role of the charge splitting process in creating the background plateau between the photo peak and the Compton continuum which is observed experimentally. At energies be- low $100 \mathrm{keV}$, the major mechanism generating the charge splitting is the carrier diffusion process, while at higher energies the transport of secondary electrons must also be considered. Indeed, at $100 \mathrm{keV}$ (Fig. 3c) the simulated and measured spectra agree only qualitatively: the photopeak, escape peak and Compton continuum features are correctly reproduced, but the simulated spectrum gives a reduced low-energy background intensity compared to that measured experimentally. This is discussed further in Section 5.

\subsection{Beam scanned across the inter-strip gap}

A virtual $60 \mathrm{keV}$ beam was scanned across the detector in steps of $10 \mu \mathrm{m}$, beginning at the contact strip edge (position $75 \mu \mathrm{m}$ from the contact strip centre) and moving across the inter-strip isolation gap. The spectrum at the contact strip was computed for each beam position and the results are shown in Fig. 5a for positions 85, 95, 105 and $115 \mu \mathrm{m}$ from the contact strip centre. The simulated spectra are in qualitative agreement with those experimentally measured shown in Fig. 5b, with the mean energy of a broad 'bump' feature moving in accordance with the beam position. This bump artefact is due to the charge splitting effect as described in Section 3.3: the contact strip closest to the incident beam receives less and less charge for each photon absorbed within the interstrip gap region as the beam is moved further from the contact strip centre. As a result, the bump in the corresponding energy spectrum shifts towards the lower energy values.

\section{Effects of electron transport}

An additional set of simulations was carried out to study the effect of electron transport in the computed spectra. Part I of the simulation program was modified by including in the EGS4 code the PRESTA routine [11] to include low-energy electron transport into the calculation of the absorbed photon history. Comparing computed spectra with and without the PRESTA routine we found no appreciable difference in our data for the case of the $\mathrm{X}$-ray beam incident on the detector contact strip 

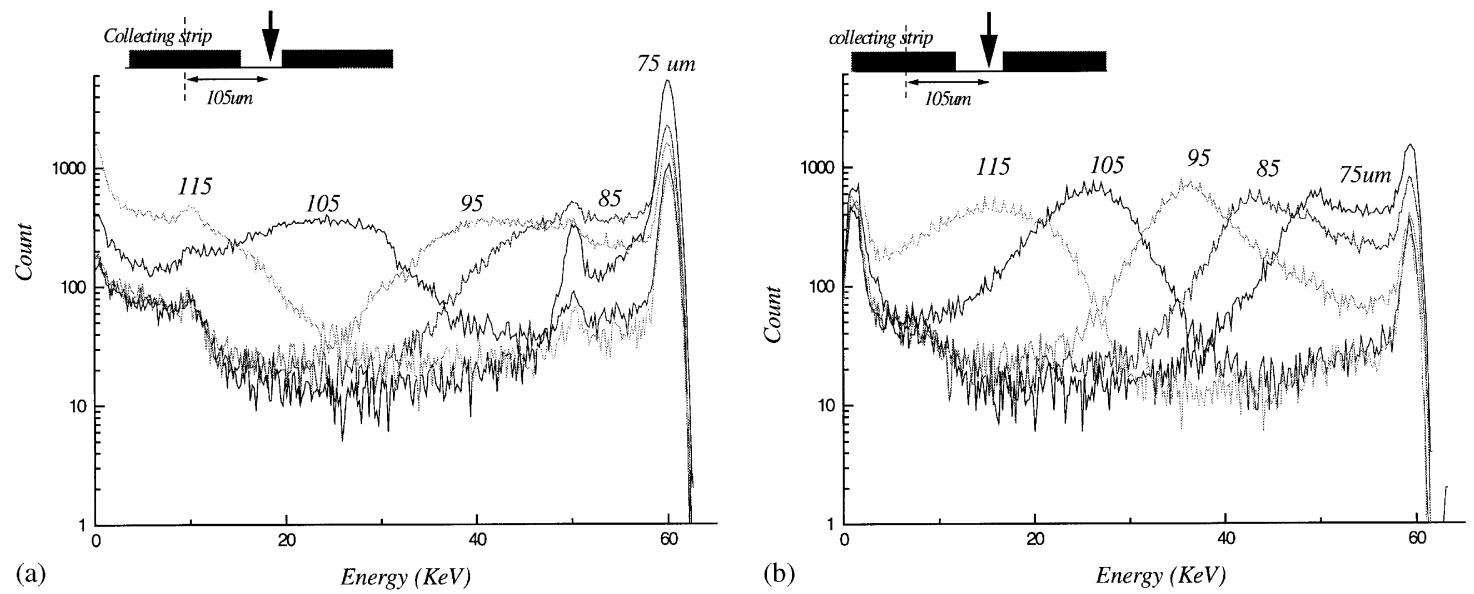

Fig. 5. (a) Simulated and (b) measured spectra at $60 \mathrm{keV}$ for a $10 \mu \mathrm{m}$ beam scanned across the interstrip isolation gap. Values 75 , 85 , 95 , 10,115 corresponds to beam centre distance in $\mu \mathrm{m}$ from the centre of the adjacent charge collecting contact strip.

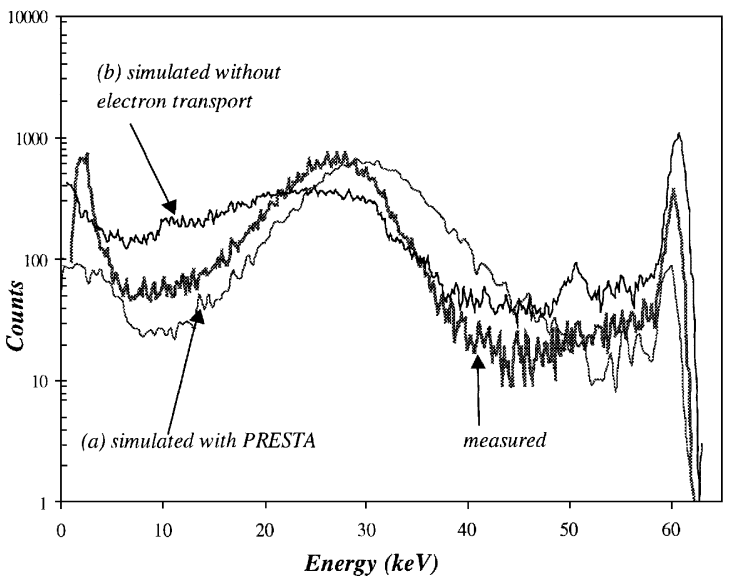

Fig. 6. Simulated and measured spectra at $60 \mathrm{keV}$ for the $10 \mu \mathrm{m}$ beam positioned above the interstrip isolation gap. Spectra are (a) with electron transport using PRESTA; and (b) with no correction for electron transport.

centre for energies below $100 \mathrm{keV}$. However, with the beam impinging incident on the interstrip gap and for energies higher than $60 \mathrm{keV}$, the use of the PRESTA routine gave spectra more better matched to those measured experimentally (Fig. 6). This is good evidence of the significance of electron transport to charge splitting between adjacent strips for energies above $60 \mathrm{keV}$ with the strip dimensions of our particular detector.

\section{Conclusion}

We have demonstrated with a simple charge diffusion model, and using the EGS4 code with the LSCAT low-energy extension, it is possible to accurately simulate experimental spectra from a germanium micro-strip detector over the photon energy range $35-100 \mathrm{keV}$. This covers a large part of the energy spectrum encountered at new, 3rd generation synchrotron X-ray sources such as the ESRF. No free parameters are used in the simulation code, and all numerical values are computed from documented physical constants or measurements taken from a real detector. The experimentally measured response of the germanium microstrip detector can be considered ideal insofar as the limits to its performance may be simply attributed to the fundamental physical processes of charge absorption and diffusion. Our simulations confirm that low-energy photopeak tailing, and the bump artefact observed in spectra obtained with an X-ray beam incident upon the inter-strip gap, both result primarily from diffusion charge splitting. The understanding of this phenomenon indicates a means of correcting such badly distorted spectra by using coincidence measurements between neighbour strips with post-summation of the signal data, and this approach has been investigated experimentally and reported elsewhere [1,2]. 
For low-energy applications $(<30 \mathrm{keV})$ the penetration depth of the $\mathrm{X}$-rays in germanium is similar or less than the detector microstrip dimensions and charge trapping occurring in weak electric field regions at the surface must be considered [1]. In order to extend the application of our code to energies $>60 \mathrm{keV}$ the effects of electron transport must be included, and we have demonstrated that this is possible with the EGS4 code with the PRESTA routine.

\section{Acknowledgements}

We wish to thank D. Gutknecht of Eurisys Mesures for the fabrication of the germanium detector and the staff of the Optics test beamline BM5 and the Detector Group of the ESRF for preparation of the experimental measurements.

\section{References}

[1] G. Rossi, J. Morse, J.-C. Labiche, D. Protic, A.R. Owens, Nucl. Instr. and Meth. A 392 (1997) 264.

[2] G. Rossi, J. Morse, D. Protic, Energy and position resolution of germanium microstrip detectors at X-ray energies
15 to $100 \mathrm{keV}, 1997$ IEEE NSS Conference Record, November 9-15, Albuquerque, NM.

[3] W.R. Nelson, D.W.O. Rogers, Structure and operation of the EGS4 code system, in: T. Jenkins, W. Nelson, A. Rindi, A. Nahum, D. Rogers (Eds.), Monte Carlo Transport of Electrons and Photons, Plenum Press, New York, 1989, pp. 287-306. See also: W.R. Nelson, H. Hirayama, D.W.O. Rogers, The EGS4 Code System. Report SLAC-265, Stanford Linear Accelerator Center, Stanford, CA, 1985.

[4] Y. Namito, S. Ban, H. Hirayama. LSCAT: low-energy photon-scattering expansion for the EGS4 Code, KEK Internal Report 95-10, August 1995.

[5] Y. Namito, S. Ban, H. Hirayama, Nucl. Instr. and Meth. A 349 (1994) 489-494.

[6] Y. Namito, S. Ban, H. Hirayama, Nucl. Instr. and Meth. A 322 (1993) 277.

[7] The passage of fast electron through matter, in: R.D. Birkhoff (Ed.), Encyclopaedia of Physics, Vol. XXXIV, S. Flugge (Ed.), Corpuscles and Radiation in Matter II, 1958.

[8] G. F. Knoll (Ed.), Radiation Detection and Measurement, 2nd Edition, Wiley, New York, 1989, p. 398.

[9] IDL: Interactive Data Language by Research Systems Inc. 2995 Wilderness Place, Boulder, CO 80301, USA.

[10] S.M. Sze, Physics of semiconductor devices, 2nd edition (formula 107), Wiley, New York, 1981, p. 55.

[11] W.R. Nelson, D.W.O. Rogers, Structure and operation of the EGS4 code system, in: T. Jenkins, W. Nelson, A. Rindi, A. Nahum, D. Rogers (Eds.), Monte Carlo Transport of Electrons and Photons, Plenum Press, New York, 1989 (Chapter 5). Electron step-size artefacts and PRESTA (A.F. Bielajew and D.W.O. Rogers). 